\title{
Clinical Status of Patients with Coronary Artery Disease Post COVID-19
}

\author{
Mukhlisa Rakhmatillaevna Khidoyatova \\ Ph.D., Associate Professor of the Department of Functional Diagnostics of the Center for the Development of \\ Professional Qualifications of Medical Workers of the Ministry of Health of Uzbekistan \\ Corresponding author email: khidoyatova.m@mail.ru
}

\section{Ulugbek Karimovich Kayumov}

Doctor of Medical Sciences, Professor, Head of the Department of Internal Diseases No. 1, Center for the Development of Professional Qualifications of Medical Workers of the Ministry of Health of Uzbekistan Email: kayumov.u@mail.ru

Feruza Khidoyatovna Inoyatova

Doctor of Biological Sciences, Professor of the Department of Medical and Biological Chemistry, Tashkent Medical Academy

Email: firuza.inoyatova.49@mail.ru

\section{Khurshid Gayratovich Fozilov}

Candidate of Medical Sciences, Director of State Institution, Republican Specialized Scientific and Practical Medical Center for Cardiology

Email: info@cardiocenter.uz

\section{Gulnoz Abdusattarovna Khamidullaeva}

Doctor of Medical Sciences, Professor, Deputy Director for Science, Head of the Department of Arterial Hypertension of the Republican Specialized Scientific and Practical Medical Center for Cardiology

Email: gulnoz0566@mail.ru

\author{
Aziz Sagdullaevich Eshpulatov \\ Head of the Department endovascular surgery of the Republican Specialized Scientific and Practical Medical Center \\ for Cardiology \\ Email:dr.azizbek84@mail.ru
}

\begin{abstract}
The main goal of our study was to identify the activity of cardiac dysfunction based on the analysis of the main cardiological methods of research, such as ECG, echocardiography, 24-hour ECG monitoring in conjunction with laboratory parameters in patients with coronary artery disease (CAD) who underwent mild and moderate COVID-19, without signs of residual effects of lung tissue damage (fibrotization). 52 patients with coronary artery disease were examined, which were divided into 2 groups depending on the past infection in history: 1 group without COVID-19 in history ( $n=26$ ) (based on history and results of SARS-CoV-2 antibody titer), 2 a group with a history of COVID-19 (n=26), confirmed by relevant documents (tests), but without oxygen therapy and steroids, in order to avoid the influence of a serious illness and drug exposure. Conclusions: dynamic monitoring of hemostasis parameters after the hospital stage in patients with CAD should be carried out in order to prevent adverse cardiovascular outcomes, even with a history of moderate and mild coronavirus infection. One of the aspects of therapeutic rehabilitation in the post-COVID period in patients with IHD is the use of vitamin D preparations.
\end{abstract}

Keywords---artery disease, clinical status, COVID-19, lung tissue damage, SARS-CoV-2 


\section{Introduction}

Data on the long-term results of COVID-19 treatment began to appear in the literature in the form of clinical cases and in a small sample of patients, however, there is evidence of the development of adverse cardiovascular outcomes after stopping an acute process in the midst of the COVID-19 clinic and during the rehabilitation period ( Akhmerov \& Marbán, 2020). One of the main reasons for the severe course of cardiovascular diseases (CVD) and the development of complications is also a violation of the hemostasis system (Raxmatillaevna \& Karimovich, 2021). Short and long-term prognosis remains an understudied aspect in patients with coronary heart disease (CHD) who have undergone COVID-19. There are enough studies in the literature that present the role of inflammatory mediators in the pathogenesis of CVD (Lubrano \& Balzan, 2015; Pasceri et al., 2000; Siti et al., 2015). A proinflammatory effect of C-reactive protein (CRP) on the vascular wall has been shown, which increases the formation of other inflammatory mediators, adhesion molecules on the endothelial surface (Lubrano \& Balzan, 2015; Siti et al., 2015). Under conditions of SARS-CoV-2 infection, the level of CRP closely correlates with the severity of the course, the volume of affected lung tissue according to computed tomography (CT), progression, and prognosis of COVID-19 (Chikina et al., 2020). The extent to which these changes are expressed in the post-COVID period in patients with CVD is also a topical issue.

In patients with CVD, special vigilance should be exercised against the background of COVID-19 infection, paying equal attention not only to the treatment of the infection but also to the control of risk factors for cardiovascular disease. CVD patients with COVID-19 are at high risk of destabilization due to a combination of stressors, systemic infection, and inflammation. Early studies have shown that people with comorbid conditions such as hypertension, diabetes mellitus suffer increased morbidity and mortality from COVID-19 (Chen et al., 2020; Wang et al., 2020). At the same time, mortality from COVID-19 increases up to five times in people with CVD (Wu \& McGoogan, 2020). Clinical and laboratory symptoms of heart damage are detected with COVID-19 quite often; some patients reported chest pain and palpitations even in the absence of typical respiratory symptoms (Shchikota et al., 2020). All this requires maximum cardiological vigilance in the treatment of patients with COVID-19, long-term observation, and timely use of various diagnostic methods in order to prevent early and late cardiovascular complications (CVC).

\section{Materials and Methods}

The study was conducted on the basis of the Republican Specialized Scientific and Practical Center for Cardiology. 52 patients with coronary artery disease were examined, which were divided into 2 groups depending on the past infection in history: 1 group without COVID-19 in history $(n=26)$ (based on history and results of SARS-CoV-2 antibody titer), 2 a group with a history of COVID-19 ( $n=26)$, confirmed by relevant documents (tests), but without oxygen therapy and steroids, in order to avoid the influence of a serious illness and drug exposure (Sattar et al., 2020; Todurov et al., 2021). The main goal of our study was to identify the activity of cardiac dysfunction based on the analysis of the main cardiological research methods, since electrocardiography (ECG), echocardiography (ECHOCG), 24-hour ECG monitoring in conjunction with laboratory parameters in patients with coronary artery disease who underwent mild and moderate COVID-19, without signs of residual effects of lung tissue damage (fibrotization). The period from the acute period of COVID-19 ranged from 2 weeks to 3 months. All subjects had anginal pain, accompanied by dynamic ECG changes, and there were no manifestations of respiratory failure. The exclusion criterion was a history of diabetes mellitus.

In order to identify the features of the course of coronary artery disease in the examined, an analysis of the clinical status was carried out, including the main indicators of ECG and hemodynamics (Table 1)

Table 1

Clinical status of CAD patients with and without COVID-19 in history

\begin{tabular}{lccccc}
\hline Indicators & \multicolumn{2}{c}{1 group $(\mathrm{n}=26)$} & \multicolumn{2}{c}{2 group $(\mathrm{n}=26)$} & $\mathrm{p}$ \\
\cline { 2 - 4 } & $\mathrm{M}$ & $\mathrm{m}$ & $\mathrm{M}$ & $\mathrm{m}$ & $\mathrm{p}$ \\
\hline Age, years & 60,15 & 1,64 & 58,42 & 1,68 & 0,464 \\
BMI & 27,12 & 0,60 & 30,61 & 0,81 & $0,001^{*}$ \\
Obesity experience (years) & 10,94 & 0,67 & 12,35 & 0,99 & 0,247 \\
AG experience (years) & 7,78 & 0,65 & 9,70 & 0,87 & 0,084 \\
CVD experience (years) & 6,32 & 0,79 & 7,92 & 1,03 & 0,223 \\
\hline
\end{tabular}




\begin{tabular}{lccccc}
\hline SpO2, \% & 96,04 & 0,26 & 95,85 & 0,25 & 0,596 \\
SBP, mmHg & 131,15 & 4,24 & 130,77 & 4,61 & 0,951 \\
DBP, mmHg & 83,08 & 1,82 & 82,31 & 1,50 & 0,746 \\
HR on ECG & 71,27 & 2,45 & 73,08 & 2,74 & 0,625 \\
P, ms & 0,10 & 0,00 & 0,10 & 0,00 & 1,000 \\
P-Q, ms & 0,15 & 0,00 & 0,16 & 0,00 & 0,611 \\
QTs, ms & 0,35 & 0,01 & 0,37 & 0,01 & $0,005^{*}$ \\
\hline
\end{tabular}

Note: $*$-significance of differences, $\mathrm{p}<0.05$

Abbreviations: BMI-body mass index; AG-arterial hypertension; CVD-cardiovascular disease; SBP-systolic blood pressure; DBP - diastolic blood pressure, HR - heart rate.

In the age aspect, the patients of the studied groups did not differ. The mean age was $60.15 \pm 1.64 \& 58.42 \pm 1.68$ years in the 1st and 2nd groups, respectively. As can be seen from the table, a significant difference was noted in the body mass index, which was higher in patients with a history of coronavirus infection. When deciphering the main ECG parameters, lengthening of the corrected Q-T interval (Q-Tc) was noted in patients of group 2, although the average values of this interval were within the reference value (Heusch et al., 2014; Mitrani et al., 2020). Cardiological characteristics of patients are presented in Table 2. In both groups, more than $80 \%$ had a history of arterial hypertension, however, at the time of admission, high levels of SBP and DBP were recorded in single patients. The average levels of SBP and DBP in the examined groups did not differ (Table 1), although a more detailed analysis showed that in patients who had a moderate course of COVID-19, the minimum level of SBP was $140 \mathrm{~mm} \mathrm{Hg}$.

Table 2

Cardiac characteristics of patients

\begin{tabular}{llll}
\hline Parameter n/\% & 1 group $(\mathrm{n}=26)$ & 2 group $(\mathrm{n}=26)$ & $\mathrm{p}$ \\
\hline AMI / with Q wave/ without Q wave & $6(23,1 \%) / 2(7,7 \%) / 4(15,4 \%)$ & $1(3,8 \%) /-/ 1(3,8 \%)$ & $0,009^{*}$ \\
History of stenting & $2(7,7 \%)$ & $1(3,8 \%)$ & $>0,05^{*}$ \\
Cardiac arrhythmias, according to ECG & $9(34,6 \%)$ & $3(11,5 \%)$ & $0,001^{*}$ \\
\hline
\end{tabular}

Note: *-significance of differences $\mathrm{p}<0.05$

Abbreviations: AMI-acute myocardial infarction

6 patients (23.1\%) who had undergone COVID-19 were hospitalized with the diagnosis of AMI, in group 1 (without COVID-19) only in one case AMI was registered. When evaluating the main indicators of ECHOCG, no significant differences were found in the studied groups (Table 3). We can only note a trend towards a decrease in SV in patients who underwent COVID-19 (n.d.).

Table 3

EchoCG parameters in the studied groups

\begin{tabular}{|c|c|c|c|c|c|}
\hline \multirow{2}{*}{ Parameters } & \multicolumn{2}{|c|}{1 group $(n=26)$} & \multicolumn{2}{|c|}{2 group $(n=26)$} & \multirow[b]{2}{*}{$\mathrm{p}$} \\
\hline & $\mathrm{M}$ & $\mathrm{m}$ & $\mathrm{M}$ & $\mathrm{m}$ & \\
\hline BSA & 1,97 & 0,03 & 1,98 & 0,03 & 0,904 \\
\hline TPWLV sm & 1,00 & 0,02 & 0,98 & 0,02 & 0,325 \\
\hline TIVS mm & 1,02 & 0,02 & 1,01 & 0,02 & 0,750 \\
\hline MMLV, $\mathrm{g}$ & 188,57 & 7,26 & 187,27 & 7,63 & 0,902 \\
\hline IMMLV, $\mathrm{g} / \mathrm{sm} 2$ & 95,98 & 3,71 & 93,15 & 3,46 & 0,579 \\
\hline $\mathrm{SV}, \mathrm{ml}$ & 66,88 & 2,32 & 63,26 & 3,17 & 0,360 \\
\hline $\mathrm{EF}, \%$ & 59,17 & 0,77 & 58,89 & 1,16 & 0,840 \\
\hline EDS LV, sm & 5,01 & 0,09 & 5,05 & 0,10 & 0,728 \\
\hline EDV LV, $\mathrm{ml}$ & 109,50 & 6,55 & 109,04 & 6,77 & 0,961 \\
\hline IEDV LV & 55,30 & 3,12 & 54,51 & 3,04 & 0,858 \\
\hline ESI LV, sm & 3,55 & 0,11 & 3,56 & 0,12 & 0,959 \\
\hline $\mathrm{ESV}, \mathrm{ml}$ & 45,71 & 4,12 & 46,10 & 4,22 & 0,947 \\
\hline LA, sm width & 3,46 & 0,09 & 3,46 & 0,09 & 1,000 \\
\hline LA, sm length & 4,62 & 0,09 & 4,52 & 0,10 & 0,479 \\
\hline
\end{tabular}




\begin{tabular}{llllll}
\hline Square & 16,13 & 0,72 & 15,79 & 0,74 & 0,744 \\
Ao, sm & 3,10 & 0,12 & 3,11 & 0,12 & 0,982 \\
RWT LV, unit & 0,41 & 0,01 & 0,40 & 0,01 & 0,517 \\
RV ave. & 2,75 & 0,08 & 2,73 & 0,08 & 0,894 \\
RV base & 3,26 & 0,07 & 3,25 & 0,07 & 0,970 \\
RA sm width & 3,18 & 0,07 & 3,22 & 0,07 & 0,737 \\
RA sm length & 4,39 & 0,07 & 4,37 & 0,07 & 0,831 \\
Wave E [m/s] & 0,66 & 0,03 & 0,66 & 0,04 & 0,963 \\
Wave A [m/s] & 0,78 & 0,03 & 0,76 & 0,03 & 0,724 \\
E/A & 0,87 & 0,06 & 0,91 & 0,06 & 0,709 \\
SP PA & 25,50 & 1,46 & 24,31 & 1,51 & 0,572 \\
\hline
\end{tabular}

Abbreviations: BSA - body surface area; TPWLV-thickness of the posterior wall of the left ventricle; TIVSthickness of the interventricular septum; MMLV - mass of the myocardium of the left ventricle; IMMLV - MMLV index; SV - stroke volume; EF - ejection fraction; EDS LV- end-diastolic size of the left ventricle; EDV - enddiastolic volume; IEDV - end-diastolic volume index; ESI - end systolic size index; ESV - end systolic volume; LA left atrium; Ao - aorta; RWT - relative wall thickness; RV- right ventricle; RA- right atrium; SP PA - systolic pressure in the pulmonary artery.

More convincing differences in cardiological parameters between the groups were obtained by us in a detailed analysis of the data of 24-hour ECG monitoring and heart rate variability. Supraventricular arrhythmias, in the form of supraventricular extrasystoles and running supraventricular tachycardias, were more frequent manifestations of arrhythmia in patients with COVID-19 than in patients without a history of COVID-19 (80.7\% vs. 46.0\%, p<0.05 ), as well as ventricular arrhythmias, in the form of ventricular extrasystoles in the second group (42.3\%) versus $15.3 \%$, $(\mathrm{p}<0.05)$. Analysis of the diurnal dynamics of QTs (Figure 1) showed higher values of the minimum (413 vs. $381 \mathrm{ms,}$ $\mathrm{p}<0.01)$, mean $(457$ vs. $\mathrm{p}<0.01)$ in patients of group 2 , compared with group 1 . Hourly analysis showed that group 2 patients had consistently longer QTs values than group 1 throughout the day (Thomas et al., 1988; Allen et al., 1978).

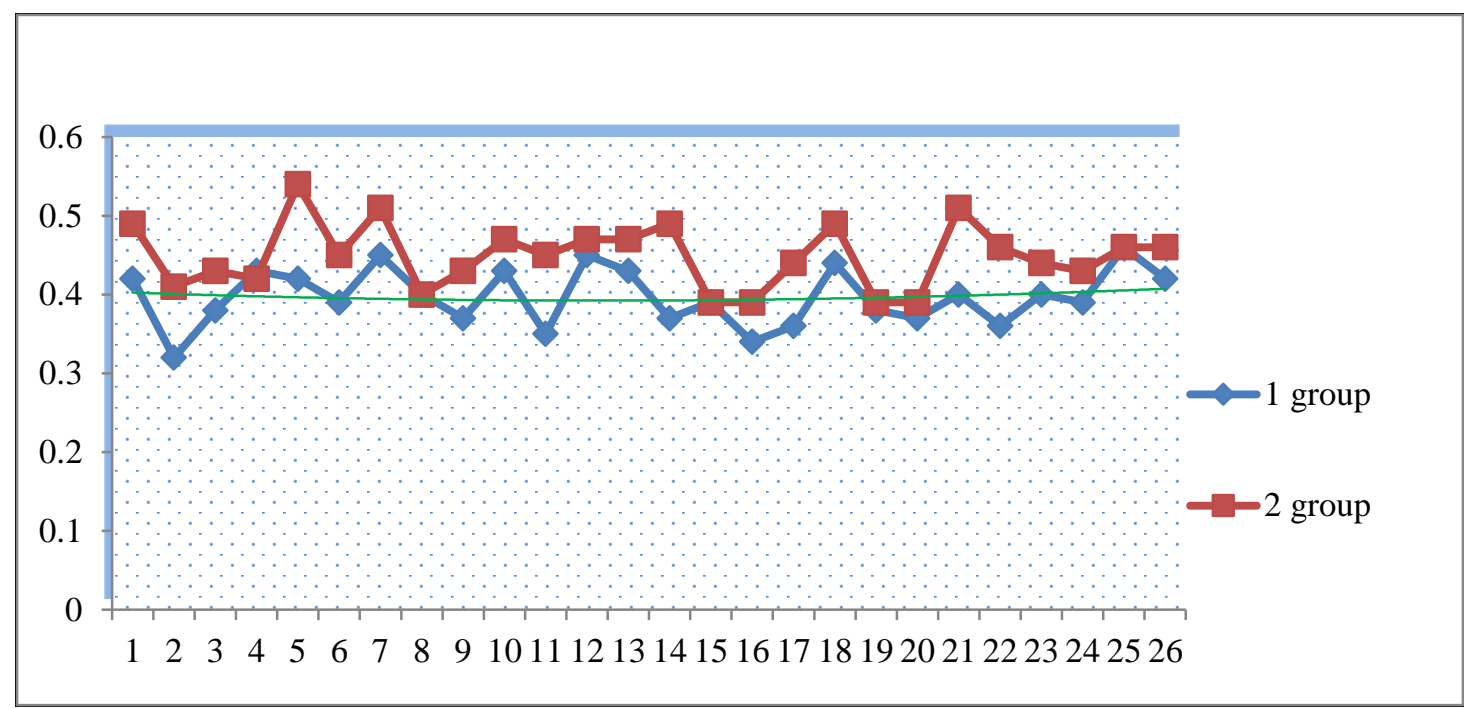

Figure 1. Average QTs parameters in examined patients based on the results of daily ECG monitoring

In patients after coronavirus infection, hyperactivation of the sympathetic nervous system (SNS) was observed, expressed in an increase in LF/HF up to 2.8 rel. units, against the background of a decrease in the activity of the parasympathetic nervous system (PSNS) (decrease in SDNNi to $38.6 \mathrm{~ms}, \mathrm{p}<0,01$ ) with a persistent disruption of circadian interactions of these parts of the autonomic nervous system, manifested in an increase in sympathetic influences on the heart rhythm and an insufficient increase in PSNS activity at night (CI LF/HF 1.1 rel. units) (Table 4). The low circadian index (CI) in the second group may have been due to the fact that patients after coronavirus infection experienced hyperactivation of the SNS at night (Scoccia et al., 2021; Angeli et al., 2021) 
Table 4

Indicators of heart rate variability in the examined patients

\begin{tabular}{lll}
\hline Parameters & 1 group $(\mathrm{n}=26)$ & 2 group $(\mathrm{n}=26)$ \\
\hline SDNNi, ms & $45,2 \pm 0,97$ & $38,6 \pm 0,85^{*}$ \\
LF/HF & 2,1 & $2,8^{*}$ \\
CI LF/HF & 1,6 & $1,1^{*}$ \\
\hline
\end{tabular}

Note: *- significance of differences $\mathrm{p}<0.05$

Abbreviation: LF/HF - Low Frequency/High Frequency ratio, CI - circadian index

Analysis of hemostasis parameters in the patients with coronary artery disease examined by us revealed a significant difference in the PTI rate of $93.8 \%$ versus $97.5 \%$ in groups 1 and 2 , respectively $(\mathrm{p}=0.041)$ (Table 5).

Table 5

Indicators of the hemostasis system and acute-phase blood proteins

\begin{tabular}{llllll}
\hline \multirow{2}{*}{ Show } & \multicolumn{2}{c}{ 1 group $(\mathrm{n}=26)$} & \multicolumn{2}{c}{ 2 group (n=26) } & $\mathrm{p}$ \\
\cline { 2 - 5 } & $\mathrm{M}$ & $\mathrm{m}$ & $\mathrm{M}$ & $\mathrm{m}$ & 0,968 \\
APTT sec. - [23,4-36,2] & 26,94 & 0,41 & 26,96 & 0,38 & 0,910 \\
INR - [0,85-1,15] & 1,04 & 0,02 & 1,04 & 0,01 & 0,124 \\
Fibrinogen g/1 - [1,8-3,5] & 2,53 & 0,08 & 2,75 & 0,11 & $0,041^{*}$ \\
PTI \% - [70-100] & 93,78 & 1,34 & 97,52 & 1,17 & 0,903 \\
PTR sec. - [13-18] & 13,92 & 0,17 & 13,89 & 0,14 & 0,872 \\
Prothrombin ratio [0,9-1,3] & 1,02 & 0,01 & 1,02 & 0,01 & $0,000^{*}$ \\
CRP mg/l - [до 5,0-(отр.)] & 2,61 & 0,12 & 4,08 & 0,31 & \\
\hline
\end{tabular}

Note: [norm]; *-significance of differences $\mathrm{p}<0.05$

Abbreviations: APTT-activated partial thrombin time; INR - international normalized ratio; PTI - prothrombin index; PTR - prothrombin ratio; CRP - C-reactive protein

It should be noted that in patients with increased body weight, a shortening of the PTT by more than $12.7 \%$ correlated with a higher level of functioning of the sympathetic nervous system ( $\mathrm{LF} / \mathrm{HF}>2.1 \mathrm{rel} . \mathrm{u})$ according to the data of daily ECG monitoring. In addition, patients with an increase in daily LF/HF>2.1 relative units had higher (by $15.2 \%$ ) hematocrit values. An inflammation marker, CRP, showed significant differences between the study groups, its concentration in group 2 was almost 1.5 times higher than in patients without a history of COVID-19 ( $p=0.000)$. In patients who recovered from COVID-19, indicators of ALT, AST, uric acid ( $\mathrm{p}>0.1$, n.d.), glomerular filtration rate (GFR), and total bilirubin ( $\mathrm{p}>0.1$, n.d.) had only an upward trend, but the level of total protein was statistically significant $(\mathrm{p}<0.001)$. Meanwhile, the levels of urea and creatinine in the blood serum also showed a slight difference between the study groups ( $p>0.1$, n.d.) (Figure 2). 


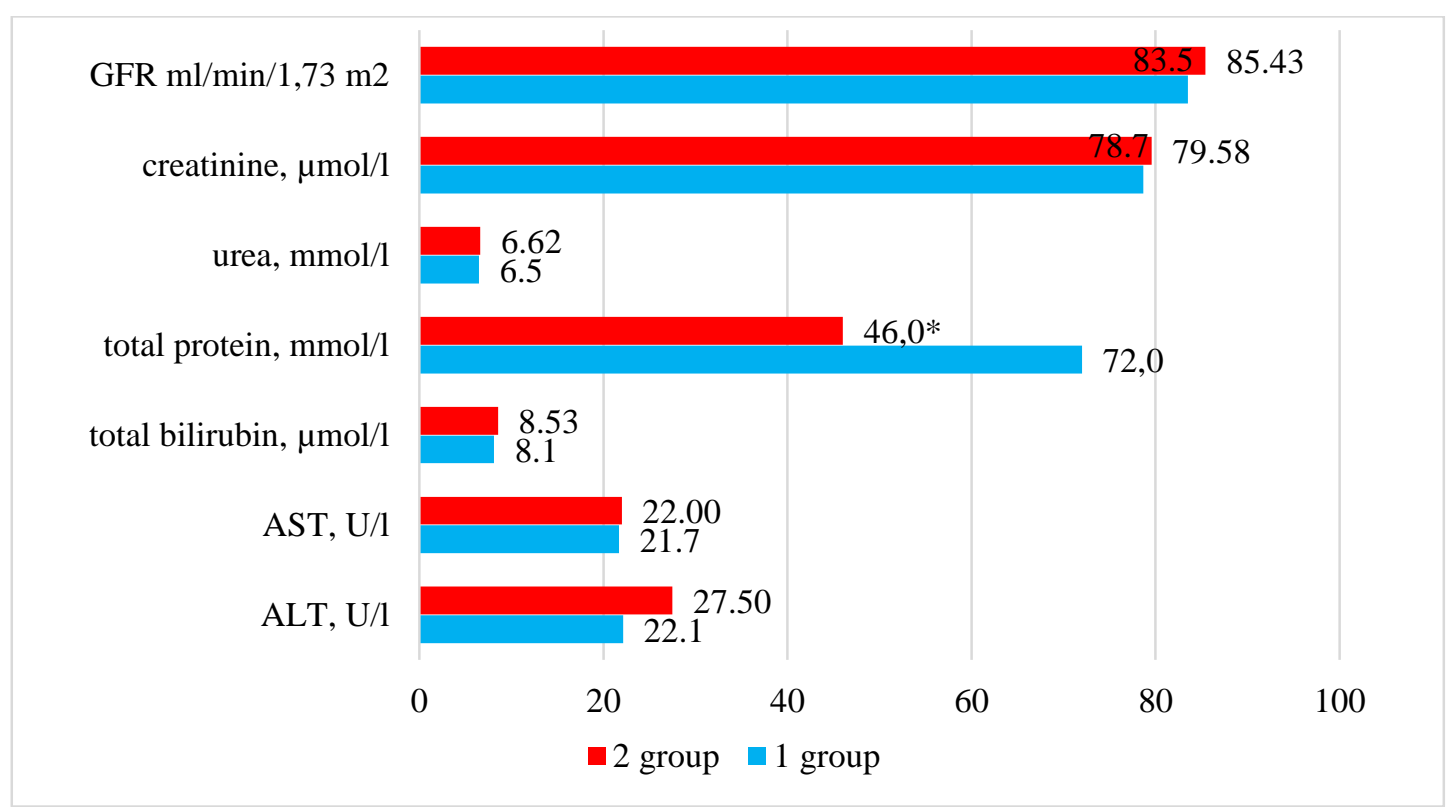

Figure 2. Biochemical parameters of the examined groups *-significance of differences $\mathrm{p}<0.05$

The level of vitamin D was significantly reduced in patients of group $2(\mathrm{p}=0.000)$. We found an inverse relationship between the level of vitamin D and the postponed period of COVID-19, i.e. the longer the period from the height of the disease, the higher the level of vitamin $\mathrm{D}$ in the blood, although it would be logical to assume lower levels in the initial periods of the post-COVID period.

\section{Discussion}

The obtained changes in the hemostasis system confirm the concept of the relationship between inflammation and thrombosis, the so-called "immunothrombosis", associated with an increase in the activation of the blood coagulation system, against the background of severe inflammation in the midst of COVID-19 and during the decline in the activity of the process (Yavelov \& Drapkina, 2020). What "dictates" further monitoring of hemostasis parameters in patients who have undergone COVID-19 in the long-term period. The concentration of CRP in patients with a history of COVID-19 was almost 1.5 times higher than in patients without a history of COVID-19 (p = 0.000), which indicates the presence of a persistent long-term residual inflammatory process even with moderate and mild coronavirus infection.

According to the results of daily ECG monitoring, the low circadian index (CI) in the second group may have been due to the fact that patients after coronavirus infection experienced hyperactivation of the SNS at night. COVID-19 leads to changes in hemostasis and contributes to hyperactivation of the SNS, a "vicious circle" is created, and both processes exacerbate each other. As a result, the thrombotic process and the risk of cardiovascular events increase. The severity of the process was associated with overweight, which may be the reason for frequent CVCs in this category of people with COVID-19. The revealed changes in vitamin D values can be explained by low levels of vitamin $\mathrm{D}$ in the acute period of the disease, as a result of infection, and a tendency to increase in the postCOVID period as a result of the restoration of the immune system. The foregoing dictates the need for the use of vitamin D preparations during the rehabilitation period (Gupta et al., 2021; Jang et al., 1994; Hussin et al., 2021).

\section{Conclusions}

- Dynamic monitoring of hemostasis parameters after the hospital stage in patients with CAD should be carried out in order to prevent adverse cardiovascular outcomes, even with a history of moderate and mild coronavirus infection.

- One of the aspects of therapeutic rehabilitation in the post-COVID period in patients with CAD is the use of vitamin D preparations. 


\section{References}

Akhmerov, A., \& Marbán, E. (2020). COVID-19 and the heart. Circulation research, 126(10), 1443-1455.

Allen, R. H., Stinson, E. B., Oyer, P. E., \& Shumway, N. E. (1978). Predictive variables in reoperation for coronary artery disease. The Journal of Thoracic and Cardiovascular Surgery,75(2), 186-192. https://doi.org/10.1016/S0022-5223(19)41285-3

Angeli, F., Marazzato, J., Verdecchia, P., Balestrino, A., Bruschi, C., Ceriana, P., ... \& Bachetti, T. (2021). Joint effect of heart failure and coronary artery disease on the risk of death during hospitalization for COVID19. European Journal of Internal Medicine, 89, 81-86. https://doi.org/10.1016/j.ejim.2021.04.007

Chen, N., Zhou, M., Dong, X., Qu, J., Gong, F., Han, Y., ... \& Zhang, L. (2020). Epidemiological and clinical characteristics of 99 cases of 2019 novel coronavirus pneumonia in Wuhan, China: a descriptive study. The lancet, 395(10223), 507-513.

Chikina, S. Y., Brovko, M. Y., Royuk, V. V., \& Avdeev, S. N. (2020). An unusual course of COVID-19 infection with late increase in C-reactive protein (clinical case reports). Pulmonologiya, 30(5), 709-714.

Gupta, Y. S., Finkelstein, M., Manna, S., Toussie, D., Bernheim, A., Little, B. P., ... \& Hota, P. (2021). Coronary artery calcification in COVID-19 patients: an imaging biomarker for adverse clinical outcomes. Clinical imaging, 77, 1-8. https://doi.org/10.1016/j.clinimag.2021.02.016

Heusch, G., Libby, P., Gersh, B., Yellon, D., Böhm, M., Lopaschuk, G., \& Opie, L. (2014). Cardiovascular remodelling in coronary artery disease and heart failure. The Lancet, 383(9932), 1933-1943. https://doi.org/10.1016/S0140-6736(14)60107-0

Hussin, D. A., Samah, M. A. A., Suhaimi, A. A., \& Kamarudin, M. K. A. (2021). A study on knowledge, attitude and practice of COVID-19 pandemic among the residents. International Journal of Health Sciences, 5(2), 177 188. https://doi.org/10.29332/ijhs.v5n2.1378

Jang, Y., Lincoff, A. M., Plow, E. F., \& Topol, E. J. (1994). Cell adhesion molecules in coronary artery disease. Journal of the American College of Cardiology,24(7), 1591-1601. https://doi.org/10.1016/07351097(94)90162-7

Lubrano, V., \& Balzan, S. (2015). Consolidated and emerging inflammatory markers in coronary artery disease. World journal of experimental medicine, 5(1), 21.

Mitrani, R. D., Dabas, N., \& Goldberger, J. J. (2020). COVID-19 cardiac injury: Implications for long-term surveillance and outcomes in survivors. Heart rhythm, 17(11), 1984-1990. https://doi.org/10.1016/j.hrthm.2020.06.026

Pasceri, V., Willerson, J. T., \& Yeh, E. T. (2000). Direct proinflammatory effect of C-reactive protein on human endothelial cells. Circulation, 102(18), 2165-2168.

Raxmatillaevna, K. M., \& Karimovich, K. U. (2021). The Relationship between the Degree of Lung Damage and Indicators of the Hemostasis System in Patients with Cardiovascular Diseases against the Background of COVID-19. Annals of the Romanian Society for Cell Biology, 6111-6117.

Sattar, Y., Ullah, W., Rauf, H., Yadav, S., Chowdhury, M., Connerney, M., ... \& Alraies, M. C. (2020). COVID-19 cardiovascular epidemiology, cellular pathogenesis, clinical manifestations and management. IJC Heart \& Vasculature, 29, 100589. https://doi.org/10.1016/j.ijcha.2020.100589

Scoccia, A., Gallone, G., Cereda, A., Palmisano, A., Vignale, D., Leone, R., ... \& Toselli, M. (2021). Impact of clinical and subclinical coronary artery disease as assessed by coronary artery calcium in COVID19. Atherosclerosis, 328, 136-143. https://doi.org/10.1016/j.atherosclerosis.2021.03.041

Shchikota, A. M., Pogonchenkova, I. V., Turova, E. A., \& Rassulova, M. A. (2020). Cardiac rehabilitation during the COVID-19 pandemic: a review of the literature. Doctor. $R u, 19$ (11), 6-11.

Siti, H. N., Kamisah, Y., \& Kamsiah, J. J. V. P. (2015). The role of oxidative stress, antioxidants and vascular inflammation in cardiovascular disease (a review). Vascular pharmacology, 71, 40-56. https://doi.org/10.1016/j.vph.2015.03.005

Thomas, E. S., Most, A. S., \& Williams, D. O. (1988). Coronary angioplasty for patients with multivessel coronary artery disease: follow-up clinical status. American Heart Journal, 115(1), 8-13. https://doi.org/10.1016/00028703(88)90511-X

Todurov, B., Bitsadze, A., \& Shorikova, D. (2021). Early postoperative complications in patients with acute myocardial infarction during emergency coronary bypassing. International Journal of Health Sciences, 5(3), 550564. https://doi.org/10.53730/ijhs.v5n3.2381

Wang, D., Hu, B., Hu, C., Zhu, F., Liu, X., Zhang, J., ... \& Peng, Z. (2020). Clinical characteristics of 138 hospitalized patients with 2019 novel coronavirus-infected pneumonia in Wuhan, China. Jama, 323(11), 10611069. 
Wu, Z., \& McGoogan, J. M. (2020). Characteristics of and important lessons from the coronavirus disease 2019 (COVID-19) outbreak in China: summary of a report of 72314 cases from the Chinese Center for Disease Control and Prevention. jama, 323(13), 1239-1242.

Yavelov, I. S., \& Drapkina, O. M. (2020). COVID-19: Hemostatic parameters and specifics of antithrombotic treatment. Cardiovascular therapy and prevention, 19(3), 2571. 